\section{(C) OPEN ACCESS}

\title{
XRCC2 mutation causes meiotic arrest, azoospermia and infertility
}

\author{
Yongjia Yang, ${ }^{1,2}$ Jihong Guo, ${ }^{1,2,3}$ Lei Dai, ${ }^{1,2,3}$ Yimin Zhu, ${ }^{1,4} \mathrm{Hao} \mathrm{Hu},{ }^{1,5}$ Lihong Tan, ${ }^{1}$ \\ Weijian Chen, ${ }^{1}$ Desheng Liang, ${ }^{1,2}$ Jingliang He, ${ }^{1,2}$ Ming Tu, ${ }^{1}$ Kewei Wang, ${ }^{1}$ \\ Lingqian $\mathrm{Wu}^{1,2}$
}

\begin{abstract}
- Additional material is published online only. To view please visit the journal online (http://dx.doi.org/10.1136/ jmedgenet-2017-105145)
\end{abstract}

${ }^{1}$ The Laboratory of Genetics and Metabolism, Hunan Children's Research Institute (HCRI), Hunan Children's Hospital, University of South China, Changsha, China

${ }^{2}$ State Key Laboratory of Medical Genetics, Central South University, Changsha, China ${ }^{3}$ Xiangya Hospital, Central South University, Changsha, China ${ }^{4}$ Hunan People's Hospital, Hunan Normal University, Changsha, China ${ }^{5}$ Department of Genetics, Maternal and Child Health Hospital of Hunan Province, Changsha, China

\section{Correspondence to}

Dr Yongjia Yang, Laboratory of Genetics and Metabolism, HunanChildren's Research Institute (HCRI), Hunan Children's Hospital, University of South China, Changsha, Hunan 410007, China;

yongjia727@aliyun.com and Dr Lingqian Wu, State Key Laboratory of Medical Genetics Central South University Changsha China;

wulingqian@sklmg.edu.cn

YY and JG contributed equally.

Received 6 November 2017 Revised 31 May 2018

Accepted 22 June 2018 Published Online First 24 July 2018

Check for updates

(C) Author(s) (or their employer(s)) 2018. Re-use permitted under CC BY-NC. No commercial re-use. See rights and permissions. Published by BMJ.

To cite: Yang Y, Guo J, Dai L, et al. J Med Genet

2018:55:628-636

\section{ABSTRACT \\ Background Meiotic homologous recombination} (HR) plays an essential role in gametogenesis. In most eukaryotes, meiotic HR is mediated by two recombinase systems: ubiquitous RAD51 and meiosis-specific DMC1. In the RAD51-mediated HR system, RAD51 and five RAD51 paralogues are essential for normal RAD51 function, but the role of RAD51 in human meiosis is unclear. The knockout of Rad51 or any Rad51 paralogue in mice exhibits embryonic lethality. We investigated a family with meiotic arrest, azoospermia and infertility but without other abnormalities.

Methods Homozygosity mapping and whole-exome sequencing were performed in a consanguineous family. An animal model carrying a related mutation was created by using a CRISPR/Cas9 system.

Results We identified a 1 bp homozygous substitution (c.41T>C/p.Leu14Pro) on a RAD51 paralogue, namely, $X R C C 2$, in the consanguineous family. We did not detect any $X R C C 2$ recessive mutation in a cohort of 127 males with non-obstructive-azoospermia. Knockin mice with Xrcc2-c.T41C/p.Leu14Pro mutation were generated successfully by the CRISPR/Cas9 method. The homozygotes survived and exhibited meiotic arrest, azoospermia, premature ovarian failure and infertility.

Conclusion $A X R C C 2$ recessive mutation causing meiotic arrest and infertility in humans was duplicated with knockin mice. Our results revealed a new Mendelian hereditary entity and provided an experimental model of RAD51-HR gene defect in mammalian meiosis.

\section{INTRODUCTION}

Infertility affects approximately $7 \%$ of men worldwide, ${ }^{1}$ and nearly $50 \%$ of infertility cases are associated with genetic defects. ${ }^{2-4}$ Azoospermia is the main cause of male infertility. To date, mutations on several genes, including SYCP $3,{ }^{5}$ NR $5 A 1,{ }^{6}$ TDRD $9{ }^{7}$ TAF4B and ZMYND15, ${ }^{8}$ TEX11, ${ }^{9} 10$ DMC1, ${ }^{11}$ TDRD $7,{ }^{12}$ MAGEB $4,{ }^{13}$ SYCE1, ${ }^{14}$ SOHLH $1^{15}$ and $M C M 8,{ }^{16}$ have been identified as the causes of azoospermia. However, the genetic basis of this defect in most infertility cases remains unknown. $^{2-412}$ Meiotic homologous recombination (HR) plays an essential role in proper chromosomal segregation in gametogenesis and enables populations to adapt during evolution by generating new combinations of DNA molecules. Two RecA-related recombinases, namely, DMC1 and RAD51, participate in meiotic HR. DMC1 is a meiosis-specific recombinase, ${ }^{17}$ whereas RAD51 is involved in mitotic and meiotic HR. ${ }^{18} 19$ Five RAD51 paralogues, namely, RAD51B, RAD51C, RAD51D, XRCC2 and XRCC3, have been reported to play indispensable roles in RAD51-mediated HR, ${ }^{20}$ but the detailed mechanism of RAD51-HR in meiosis remains unclear. Studies on RAD51-HR in meiosis are limited because the mammalian knockout of any RAD51 or RAD51 paralogues is embryonically lethal. ${ }^{21-25}$ In the present paper, we described a RAD51 paralogue point mutation that causes meiotic defects, that is, a XRCC2 recessive mutation (c.41T >C/p.Leu14Pro) leading to meiotic arrest, azoospermia and infertility in humans and mice. Given that humans and mice with XRCC2 mutation survive and show no phenotypes other than reproductive defects, we believe that c.41T $>$ C substitution affects the meiosis-specific function of the XRCC2 protein.

\section{MATERIALS AND METHODS}

\section{Subjects and clinical assessments}

This study was approved by the ethics committee of the Hunan Children's Hospital. Before the study, all participated subjects signed a written informed consent. This study included an infertile family (details described in RESULTS section) and 127 sporadic patients (five from Chinese Tujia Miao ethnicity and 122 are from Chinese Han-ethnicity) with non-obstructive azoospermia. These 127 patients were prospectively recruited at Jiahui Genetics Hospital, State Key Laboratory of Medical Genetics, Central South University, Changsha City, China (JGH-SKLMG) from January 2008 to October 2016. At the JGH-SKLMG, after all the azoospermic cases were clinically examined for the anatomical integrity of genital system, the patients who had any history of childhood disease, environmental or radiation exposure, prescription drug usage that could account for their infertility, and other pathologies as varicocele or cryptorchidism were excluded from the investigation. The patients were diagnosed with the clinical findings and several parameters: (1) the conducting of two separate semen analyses (3 weeks apart, each following 3 days of sexual abstinence, according to the WHO criteria, WHO laboratory manual for the examination of human semen and semen-cervical interaction 1999), (2) the percutaneous epididymal sperm aspiration, (3) the blood hormone analysis that included follicle stimulating hormone, luteinising hormone, prolactin and testosterone, (4) the 

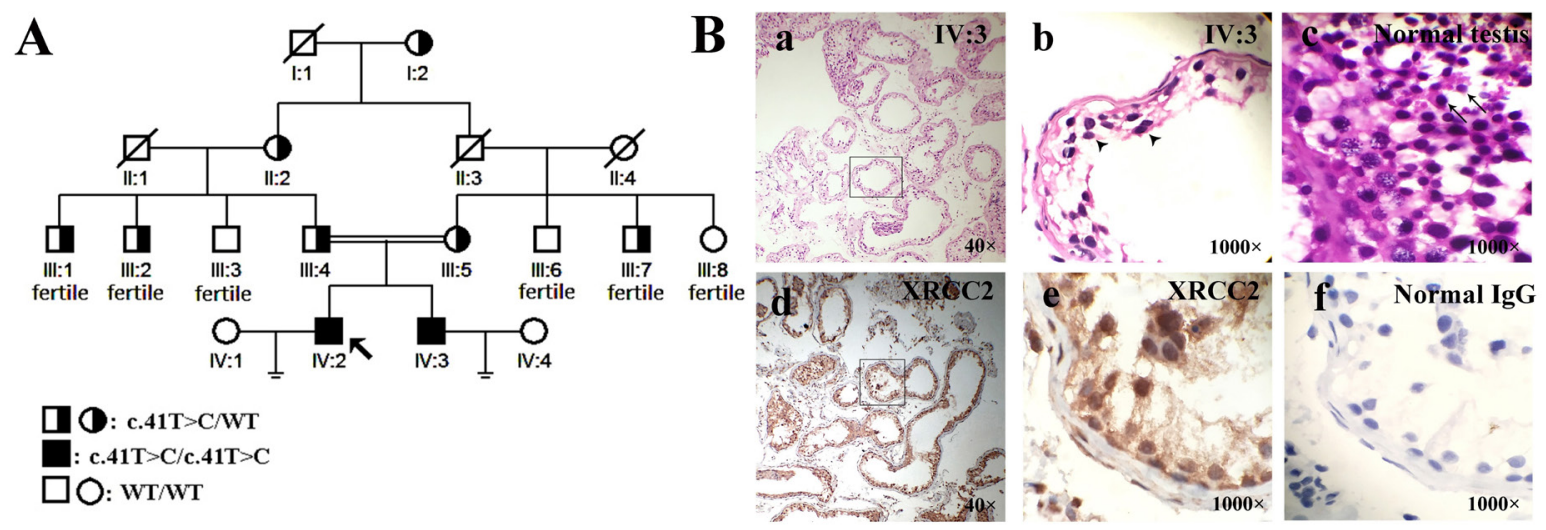

C
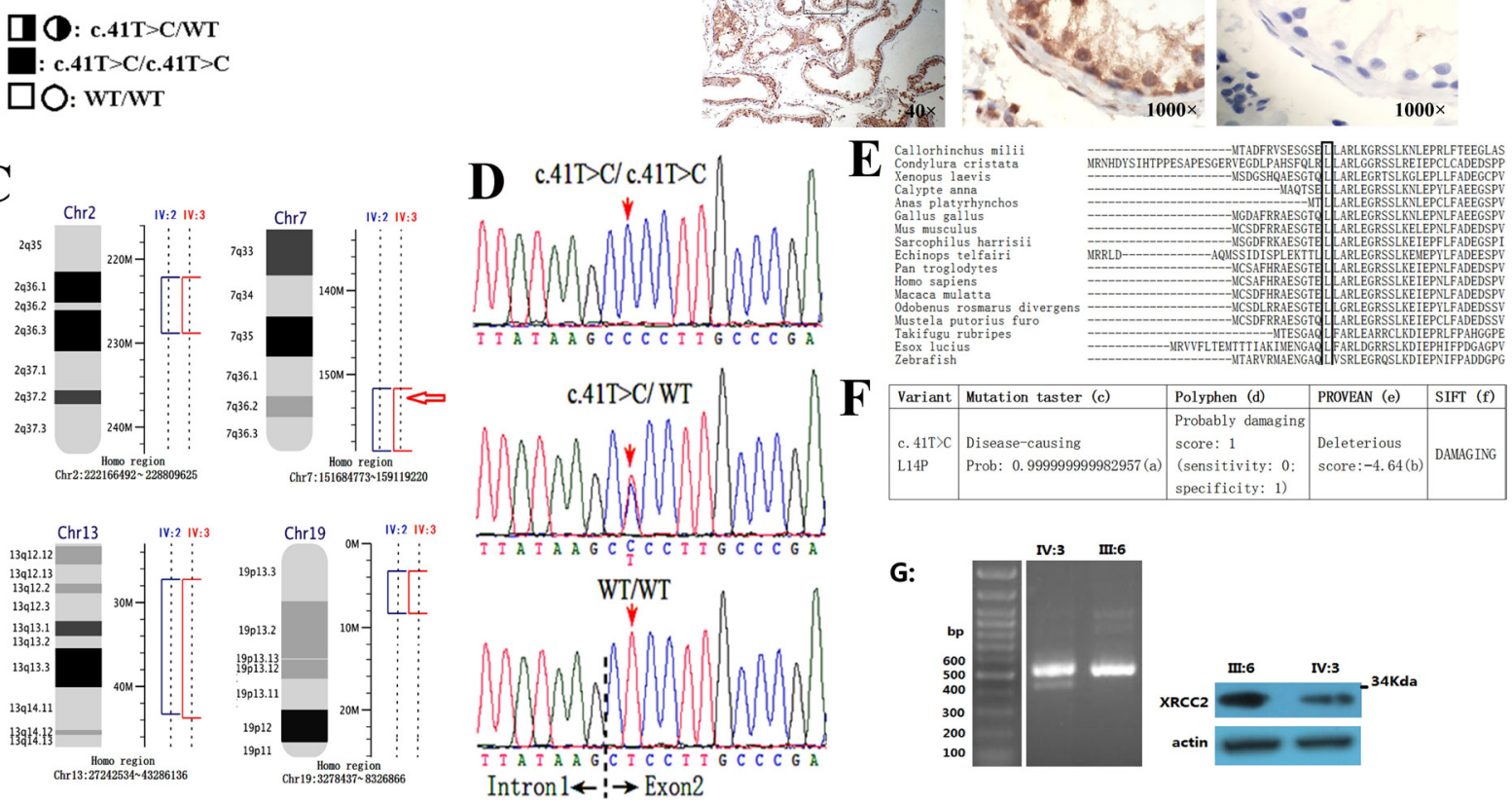

Figure 1 Mutated XRCC2 in a family with meiotic arrest, azoospermia and infertility. (A) Pedigree with XRCC2 mutation. (B) Histology of human seminiferous tubules. Magnification: $a, d=40 \times ; b, c, e, f=1000 \times$. (a,b) H\&E staining disclosed that the tubules of IV:3 (29 years old) were irregularly shaped. No mature sperm was observable, and the cells were arrested at the zygotene stage of meiosis prophase I (arrowhead). (c) H\&E staining of tubules in normal human testis (67 years old). The cells at least proliferated to the stage of round spermid (arrow). (d,e,f) Immunohistochemistry of XRCC2 in tubules of IV:3. Sections immunohistochemically labelled for XRCC2 (in brown) (d,e) or an IgG antibody as negative control (f). (C) Linkage mapping in the family disclosed the homozygous intervals shared by two patients (IV:2 and IV:3). Red arrow: Human XRCC2 position. (D) Chromatograms of XRCC2 mutations by Sanger sequencing. (E) Evolutionary conservation of the p.Leu14 residues of XRCC2 protein. (F) Functional predictions of XRCC2-p.Leu14Pro substitution: (a) Probability value mean probability of prediction, the value close to 1 indicates a high security of the prediction. (b) PREDICTION (cut-of =-2.5). (c) http://www.mutationtaster.org/. (d) http://genetics.bwh.harvard.edu/pph2/. (e) http://provean.jcvi.org/genome_submit_2.php. (f) http://sift.jcvi.org/www/ SIFT_chr_coords_submit.html. (G) RNA and protein assay on peripheral blood lymphocyte. L: Fragments amplified from the reverse transcribed cDNA sample of IV:3 and III:6 and then transferred on $0.8 \%$ agarose gel. Note: Two bands were observed in IV:3. R: Western blot detected the full XRCC2 protein on IV:3 and III:6.

karyotype analysis that was performed with G-banding methods at 550 bands level, for each karyotype analysis, at least 30 peripheral blood metaphases were analysed, (5) the microdeletions in the AZF region and the SRY gene region of the $\mathrm{Y}$ chromosome were screened by regular PCR.

\section{DNA isolation}

Peripheral-blood samples for DNA extraction were obtained from 18 family members as described in figure $1 \mathrm{~A}$ and 27 sporadic cases of non-obstructive azoospermia according to a standard protocol.

\section{Homozygosity mapping}

Whole-genome genotyping of six family members (I:2, II:2, III:4, III:5, IV:2 and IV:3 in figure 1A) was carried out to identify the presence of runs of homozygosity (ROH). Briefly, $250 \mathrm{ng}$ of genomic DNA per sample was processed on an Illumina Human Omni ZhongHua-8 Beadchip array (Illumina, San Diego,
California, USA) according to a standard protocol. Normalised output was generated using Genome Studio V.2011.1 (Illumina). The plink software (http://zzz.bwh.harvard.edu/plink/ibdibs. shtml\#homo) was used to search for $\mathrm{ROH}$ regions according to established criteria.

\section{Whole-exome sequencing}

Whole-exome sequencing (WES) was carried out for four family members (III:4, III:5, IV:2 and IV:3, figure 1A). In brief, genomic DNA (approximately $1.5 \mathrm{ug}$ ) for each member was fragmented to an average size of $350 \mathrm{bp}$ and subjected to DNA library creation using established Illumina paired-end protocols. Adaptor-ligated libraries were amplified and indexed via PCR. Then the library was hybridised to NimbleGen SeqCap EZ library Exome Capture Kit (44M, Roche, Madison, USA), and paired-end 90-base massively parallel sequencing was carried out on an Illumina HiSeq 2000, according to the manufacturer's protocols. The reads mapping and variants analysis pipeline is as follows. 
Raw reads with low quality or containing adapters were filtered before mapping. For SNP calling, filtered reads were aligned to the human genome reference (UCSC hg19) with Short Oligonucleotide Analysis Package (SOAP, V.2.21) ${ }^{26}$ and then SOAPsnp software (V.1.05) ${ }^{27}$ was used to detect SNPs. We eliminated low quality SNPs with a score of genotype quality less than 20 or the sequencing depth is smaller than 4. For indel calling, BurrowsWheeler Aligner ${ }^{28}$ was used to do the alignment. Duplicate reads were marked using the Picard toolkit and Genome Analysis Tool Kit $(\mathrm{GATK})^{29}$ was used to call small indels.

\section{Sanger sequencing}

Sanger sequencing was performed on an ABI-A3500 genetic analyzer, and a BigDye 3.1 sequencing kit (Applied Biosystems, California, USA) was used. The primers used for PCR and Sanger sequencing are provided in online supplementary table S1.

\section{Generation of $X r c c 2-c .41 T>C / p$.Leu14Pro mice}

C57BL/6 mouse model with point mutation (c. $41 \mathrm{~T}>\mathrm{C}$ ) at $\mathrm{Xrcc} 2$ locus was generated by microinjection of CRISPR/ Cas9 into fertilised eggs (Cyagen, China). The mouse Xrcc2
A

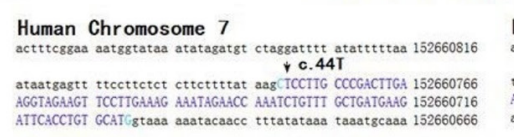

EXON 2 of $X R C C 2$ :

Mouse Chromosome 5

acataaetas tttctagaac gttgtgtcce gttaagtaat attttccccc 25698348 etttettet gtaag TCCCT TGCCCGACIT GAAGGCACAA GCTCGITGA 25698298 AGACTAGA CCCAACCTGI TTGCTGATGA AGATTCACCA GTGCACGITI 256982, aaataaccas agectgtgac agg8cggtet gtaageccet cetcraatag 2569819

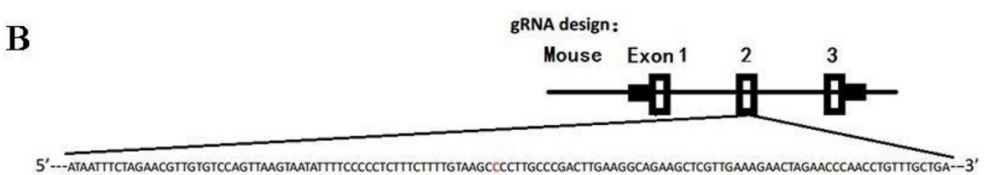

gRNA1: AAGCTCCTTGCCCGACTTGAAGG (priority) gRNA2: AAGGAGCTTACAAAAGAAAGAGG Oligo donor

ATAATTTCTAGAACGTTGTGTCCAGTTAAGTAATATTTTCCCCCTCTTTCTTTTGTAAGCCCCCTTGCCCGACTTGAAGGCAGAAGC CGITGAAAGAACTAGAACCCAACCTGTTTGCTGA

E

F

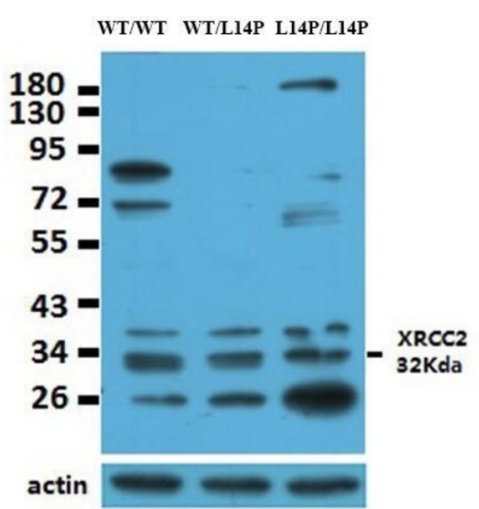

gene (GenBank accession number: NM 020570.2; Ensembl: ENSMUSG00000028933) was located on chromosome $5 \mathrm{qB} 1$. The base c.T41 mutation in oligo donor was introduced into exon 2 by homology-directed repair. Cas 9 mRNA, gRNA and oligo donor were coinjected into the zygotes for KI mouse production (figure 2A,B). The pups were genotyped by PCR and Sanger sequencing (primers and conditions, see online supplementary table S1) using the genomic DNA or cDNA as template.

To minimise the risk of off-target effects caused by CRISPR-Cas9, we used the following strategies. (1) We used a gRNA with a high score of 85 . The higher the score was, the lower the likelihood of the off-target effects would be. (2) We generated four F0 mice, namely, F2, M3, M21 and F22. Of these four F0 mice, M3 and M21 males were sterile. The two remaining F0 females (F2 and F22) were subsequently used as founders of two independent strains and backcrossed with wild type (WT) mice for two generations to produce NF2 descendants that were intercrossed to generate $\mathrm{Xrcc} 2^{\mathrm{L} 14 \mathrm{P} / \mathrm{L} 14 \mathrm{P}}, \mathrm{Xrcc} 2^{\mathrm{L} 14 \mathrm{P} / \mathrm{WT}}$ or $\mathrm{Xrcc} 2^{\mathrm{WT} /}$ WT mice. (3) We transmitted Xrcc2-c.41T $>$ C mutation in mice for six generations and found that the azoospermia phenotype
C
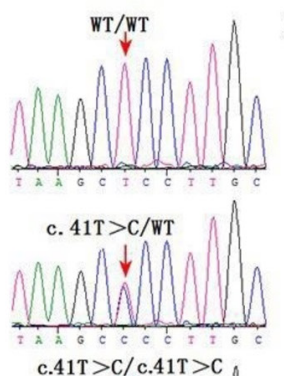

$\mathbf{G}$
WT:

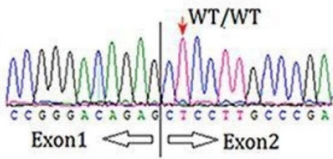

Het:

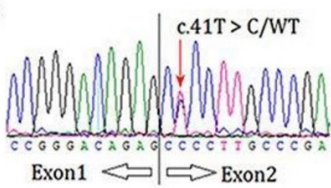

D

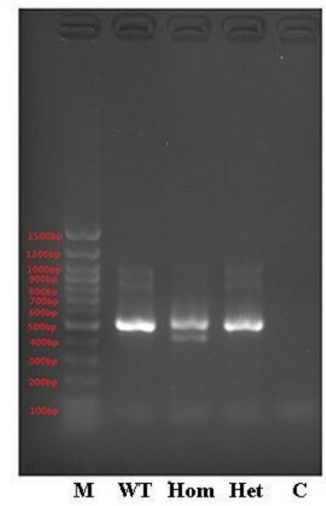

Two effects of homozygous c.41T>C: (1) Skipping the exon 2

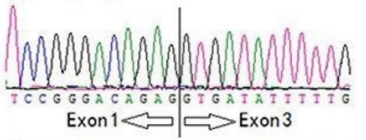

(2) c. $41 \mathrm{~T}>$ C leading to p.Leu14Pro

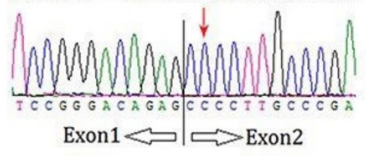

Figure 2 Generation of Xrcc2 knockin mice by CRISPR/Cas9-mediated genome engineering. (A) Genomic sequence characteristics nearing Xrcc2 exon 2. (B) Schematic of target site at mouse Xrcc2 locus. In donor DNA, the single-strand oligo-deoxynucleotide, namely, the replaced nucleotide, was observed. (C) Chromatograms of Xrcc2 mutations from PCR-amplified fragments from mouse tail genomic DNAs. (D) Fragments amplified from the reverse transcribed cDNA sample of mice testes and then immigrated on $0.8 \%$ agarose gel. Note: Two bands for homozygotes were observed. (E) Mean expression ( \pm SEM) of Xrcc 2 transcripts $(n=3)$ in three groups of pubs $(P<0.05)$. Note: approximately $70 \%$ of normal Xrcc 2 transcripts were observed on homozygous mutant testes mRNA. (F) Western blot detected the Xrcc2 proteins in all three groups of testes. Identically sized bands are detected in WT, heterozygote and mutant testis lysates, including a band at the size expected for Xrcc2, strongly suggesting the mutant Xrcc2 protein is present in the testes of homozygous mutant mice. (G) Reconfirmation of Xrcc2-c.41T>C in heterozygotes and homozygotes in the RNA level. To amplify the fragment from cDNA, the forward primer is located on exon 1, the reverse primer is located on exon 3, the sequencing primer is the forward primer. Note: For homozygote, two bands were amplified, which were sequenced individually. The results indicated the exon 2 was skipped for some of the Xrcc2 transcripts. Note: For figure CDEFG, the mice age is 90 days. WT, wild type. 
segregated with the homozygote genotype in the two Xrcc2-KI mouse lines (F2 and F22), respectively.

All the animal experiment protocols of this study were approved by the Animal Experiment Committee of Hunan Children's Hospital (Changsha, China). All experiments were performed in accordance with the approved guidelines and regulations.

\section{Histology and TUNEL assay}

Mice ovaries or testes were fixed in Bouin's solution. After dehydrated through an ethanol series, tissues were embedded in paraffin and serially sectioned, and stained with H\&E. For TUNEL assay, the In Situ Cell Death Detection Kit (Kit for immunohistochemical detection and quantification of apoptosis at single cell level, based on labelling of DNA strand breaks, Cat. 11684817910, Roche Applied Science) was used according to the manufacturer's instructions.

\section{Immunocytochemistry}

Testicular tissue sections from IV:3, which is a patient with infertility belonging to the family (figure 1A), WT mice and $X r c c 2^{\mathrm{L} 14 / \mathrm{L} 14 \mathrm{P}}$ mice were immunereacted with a primary antiXRCC2 antibody (1:100 dilution of rabbit polyclonal antibody, Gene Tex,GTX16397) to assess the XRCC2 expression in the testes. The test of affinity of the XRCC2 antibody (Gene Tex, GTX16397) was performed by over expression assay (online supplementary figure S1).

The primary antibody was detected using an HRP-labelled goat anti-rabbit IgG polymer (PV-6001, ZSGB-BIO, China). HRP interacting with hydrogen peroxide and dark brown DAB was visualised on a microscope in accordance with the product protocol.

\section{Western blotting}

Protein lysates from control and mutant testes (mice) or from peripheral blood lymphocyte (PBL) (human) were prepared. The total protein content was determined with the Bradford method. Each sample was subjected to SDS-PAGE and the membrane was immunoblotted with rabbit XRCC2 antibody (GeneTex, GTX16397) at a 1:1000 dilution. Secondary IgG-HRP antibody (1:2000; Santa Cruz Biotechnology) and an ECL Chemiluminescence kit (GE healthcare) were used for signal visualisation.

\section{RNA extraction and real time PCR}

Total RNA was extracted from testes tissues (mice) or from PBL (human) using RNAiso Plus Kit (TaKaRa, Dalian, China). cDNA was synthesised from 1 ug of RNA using the PrimeScript II 1st Strand cDNA Synthesis Kit (TaKaRa, Dalian, China). PCR reactions were performed on a 7500 Real Time PCR System (Applied Biosystems) using NovoStart SYBR qPCR SuperMix (Novoprotein, Shanghai, China). The primers were used in the experiments of qPCR: XRCC2-5'UTR-F and XRCC2-3'UTR-R (online supplementary table S1). Each assay was duplicated four times. The data were analysed by unpaired two-tailed t tests using R software.

\section{Chromosome breakage assay}

For assays of chromosome breakage in XRCC2 ${ }^{\mathrm{L} 14 \mathrm{P} / \mathrm{L} 14 \mathrm{P}}$ cells and other cells, the PBLs were treated with mitomycin C (MMC, Catalogue\#: 2713-5; CAS Number: 50-07-7; BioVision, 155 s. Milpitas Boulevard, Milpitas, California, USA) or Cisplatin (DDP, Cas No.: 15663-27-1; Houston, Texas, USA). Except for a certain amount of DNA breaking agents (MMC or DDP) or the same volume of PBS added into the RMPI-1640 medium, all the cell-culture and experimental procedures are according to standard Giemsa banding protocol in medical genetic laboratory.

\section{RESULTS}

\section{Human clinic data}

A Tujia ethnic minority family with male infertility residing in the Xiangxi Tujia-Miao Autonomous Prefecture, a west region of Hunan Province, China, participated in this study (figure 1A). Small testes were detected in the patients (IV:2 and IV:3) at 10 and 12 years as a result of routine regular health check-ups at schools. The sizes of the testes of IV: 2 and IV:3 remained small at the ages of 29 and 31 years (about 9-11 mL), respectively. Infertility in IV:2 and IV:3 was diagnosed by two independent andrologists with the following parameters: (1) two separate semen analyses (3 weeks apart, each following 3 days of sexual abstinence) reported no sperm observed (online supplementary table S2), (2) no sperm was observed in two separate occasions of percutaneous epididymal sperm aspiration (data not shown), (3) testicular biopsy reported no sperm observed and indicated meiotic arrest (figure $1 \mathrm{~B}$ and online supplementary figure S2) and (4) normal results were reported in other clinical tests (online supplementary table S3). We found no other inherited disease in this family, and the oldest family member (I:2) is a 97-year-old woman.

\section{Homozygosity mapping narrowed down to $35.2 \mathrm{Mb}$ intervals}

Given the parental consanguinity for the proband in the family, homozygous intervals were identified (online supplementary figure S3). A total of 35.2 Mb genomic intervals (2q26.1-q36.3, 7q36.1-qter, 13q12.1-q14.1 and 19p13.2-19p13.3) shared by the two infertile brothers were identified (figure 1C). In these intervals, we did not observe any region showing overlap with the previously described loci of azoospermia, meiotic arrest and infertility. This result implies that one of them may represent a previously undescribed locus of meiotic arrest and infertility.

\section{Whole-exome sequencing (WES) revealed $X R C C 2$ recessive mutation}

WES was performed and an average of $4.97 \mathrm{~Gb}$ data was yielded within the target region of each sample. The quality statistics for WES are shown in online supplementary table S4. We found 29241 variants in III:4, 29350 variants in III:5, 29361 variants in IV:2, 29920 variants in IV:3. After merging the shared variants, a total of 40728 variants were obtained in this family. First, only variants with allele frequency less than $1 \%$ in population were considered for subsequent analysis. After screening and filtering the following databases, that is, gnomAD_exome ALL, gnomAD_exome_EAS, gnomAD_genome_ALL, gnomAD_ genome_EAS, Freq_ExAC_ALL, Freq_esp6500siv2_all and Freq_1000g2015aug_all, only 2441 variants were left. We then limit the variants in coding/splicing regions (removing intergenic, intronic, upstream and downstream variants), the number of variants was reduced to 1968 . After removing benign or likely benign variants predicted by InterVar, ${ }^{30} 1444$ variants were left. Among the left variants, only 10 homozygous variants were shared by the two azoospermic men (online supplementary table S5). Further excluding the variants that are homozygous in either parent, only one variant was left (online supplementary table S6).

The brief filter strategy was provided in online supplementary figure S4. 
This variant was a 1 bp substitution on XRCC2 (c.41T $>$ C; Chr7:152357866 T>C). Coincidentally, XRCC2 gene is located in the 7q36.1-qter homozygous interval (figure 1C).

\section{$X R C C 2$ sanger sequencing and functional analysis}

Sanger sequencing confirmed the XRCC2-c.41C $>$ Tmutation and showed that it cosegregated with azoospermia in the family (figure 1A). In accordance with the reference sequence (NM_005431), the c.41T $>$ C changed codon 14 from CTC to $\mathrm{C} \bar{C} \mathrm{C}$ (figure 1D), resulting in the substitution of leucine by proline (p.Leu14Pro).

Multiple alignment analysis results indicated that residue p.Leu14 was highly conserved among different species (figure 1E). Consistently, functional prediction with four programmes in silico implied that XRCC2- p.Leu14Pro was disease-causing or damaging (figure $1 \mathrm{~F}$ ). Meanwhile, we noticed that the c.41T $>$ C substitution neared the splicing site of exon 2 of the XRCC2 gene (figure 1D).

We attempted to verify whether the XRCC2 protein is still present in the patients' testes. In seminiferous tubules from the IV:3 patient, the XRCC2 protein was detected by histological analysis (figure $1 \mathrm{Bd}-\mathrm{f}$ ). Total RNA and protein were extracted from PBLs. Consistently, we observed that the full XRCC2 transcripts and the full XRCC2 protein can be detected on extracts of PBLs (figure 1G). XRCC2 is an HR and double-strand break (DSB) repair gene. ${ }^{22} 243132$ Latest research shows atypical Fanconi anaemia and a marked increase in the frequency of chromosomal DSBs in response to crosslinking agents on a child with XRCC2 truncating mutation. ${ }^{31} 32$ Accordingly, we quantified the chromosomal breaks induced by two chromosome crosslinking agents, namely, MMC or cisplatin (DPP), on cells from IV:2 and IV:3. However, in lymphocytes cultured with MMC or DPP, the XRCC2-p.L14P cells exhibited similar frequencies of chromosomal breaks to those of normal cells (online supplementary table S7 and figure S5). Simultaneously, we did not find other phenotypes in the family.

To confirm that the XRCC2 mutation caused human meiotic arrest and azoospermia, we performed direct sequencing of XRCC2-coding regions on 127 infertile men with non-obstructive azoospermia. However, none of them carried the c.41T > C mutation or other XRCC2- recessive mutation (data not shown). We then proceeded with the generation of the animal model.

\section{Generation of $X r c c 2-c .41 T>C / p$.Leu14Pro mice}

We observed high similarities between human XRCC2 and mice Xrcc2 genomic sequences (figure 2A). The CRISPR/Cas9 method was used to generate knockin mice with the Xrcc2-c.41T>C/p. Leu14Pro mutation (below described $X r c c 2^{\text {L14P }}$ ) when the genotypes were Sanger-sequenced at genomic DNA (figure 2C) or mRNA levels (figures 2D, E and G). At the mRNA level, we designed primers that were located on the 5'UTR or 3'UTR region of mice $\mathrm{Xrcc} 2$ (online supplementary table S1). When mRNA was extracted from the mice testes, Xrcc2-c.41T >C was reconfirmed in heterozygotes (figures $2 D, E$ and $G$ ). In homozygotes, we observed a double effect of Xrcc2: (1) the c.41T>Csubstitution and (2) skipping of the whole exon 2 (which leading to a truncating protein p.L14Vfs*20) in about $30 \%$ of the XRCC2 transcripts (figures 2D, E and G).

$X r c c 2^{\mathrm{L} 14 \mathrm{P} / \mathrm{WT}}$ mice intercrosses transmitted the mutation to their offspring at normal Mendelian frequencies (online supplementary table S8, 21 litters, 188 offspring calculated, wt $=46$, heterozygote $=99$ and homozygote $=43$ ).
No difference in weight was found among the three groups of mice at 7, 30 and 60 days postpartum (dpp; online supplementary tables S8-12). Morphologically, the WT, $X r c c 2^{\mathrm{L} 14 \mathrm{P} / \mathrm{WT}}$, and $X r c c 2^{\mathrm{L} 14 \mathrm{P} / \mathrm{L} 14 \mathrm{P}}$ animals were indistinguishable at $7,14,28$ or $35 \mathrm{dpp}$ (representative see online supplementary figure S6).

\section{All of the male $X r c c 2^{\text {L14P/L14P }}$ mice exhibited meiotic arrest, azoospermia and infertility}

The male phenotype of $X r c c 2^{\mathrm{L} 14 \mathrm{P} / \mathrm{L} 14 \mathrm{P}}$ mice was analysed. We monitored 8-week-old Xrcc2 $2^{\text {L14P/L14P }}$ males mated with WT mice for 60-90 days and observed that 100\% of homozygotes were infertile (online supplementary table S13) ( $n=17)$. Afterwards, 28 male homozygotes were further evaluated, and all of the homozygotes were infertile. The infertile Xrcc2 $2^{\text {L14P/L14P }}$ males $(n=10)$ were sexually active, and this finding was indicated by the repeated detection of vaginal plugs in the mating females. The testes of the $\mathrm{Xrcc} 2^{\mathrm{L} 14 \mathrm{P} / \mathrm{L} 14 \mathrm{P}}$ mice were smaller than those of the WT mice from $21 \mathrm{dpp}$ to $180 \mathrm{dpp}(\mathrm{p}<0.05)$. In contrast to WT males weighing 97-104.5 mg (figures 3A and 7 ), the $X r c c 2^{\text {L14P/L14P }}$ mice weighed $27.3-37.9 \mathrm{mg}$ at 90 dpp. The histological examination of the testes from $90 \mathrm{dpp}$ $X r c c 2^{\mathrm{L} 14 \mathrm{P} / \mathrm{L} 14 \mathrm{P}}$ males revealed the following results: (1) nearly all of the seminiferous tubules were depleted (figure $3 \mathrm{Bc}-\mathrm{f}$ ); (2) no mature sperm was observed in all of the tubules (figure 3Bc-f); (3) in each testis, the tubules showed multiple layers of spermatocytes arrested in the zygotene and pachytene stages of meiotic prophase I (figure 3Bef and S6ef at 28 or $180 \mathrm{dpp}$ mice) and (4) the TUNEL assay indicated that the number of apoptotic spermatocytes markedly increased in some tubules (figure $3 \mathrm{C}$ ). The tubules were histologically examined at 7, 14, 21, 28, 90 and $180 \mathrm{dpp}$. However, no sperm was detected in seminiferous tubules from the homozygotes from $7 \mathrm{dpp}$ to $180 \mathrm{dpp}$, indicating a complete meiotic arrest (online supplementary figure S7).

\section{Mutant XRCC2 is present in testes}

The WT Xrcc2 protein is highly expressed in testis, ${ }^{33}$ similar to the above mentioned results at mRNA lever. Consistently, we observed that the Xrcc2 protein was expressed in the inner layer of spermatocytes of mouse tubules (figure $3 \mathrm{Da}$ and $3 \mathrm{Db}$ ) and mutant XRCC2 was detected in Xrcc2 $2^{\mathrm{L} 14 \mathrm{P} / \mathrm{L} 14 \mathrm{P}}$ mouse tubules (figure $3 \mathrm{Dc}$ and $3 \mathrm{Dd}$ ). This observation was consistent with human results, which demonstrated that the mutant Xrcc2 could be visualised in mutant tubules (figure $1 \mathrm{Bd}-\mathrm{f}$ ). In Western blot analysis, the full Xrcc2 protein was present in the testis extracts from homozygotes and other animals (figure 2F).

\section{Half of the $X r c c 2^{L 14 P / L 14 P}$ females exhibited infertility, whereas the other half showed a low reproductive capacity}

We monitored 8-week-old Xrcc2 ${ }^{\text {L14P/L14P }}$ females mated with WT mice for approximately 60 days and observed that $47 \%$ of the female homozygotes were infertile (online supplementary table S14). The repeated detection of vaginal plugs indicated that the infertile $X r c c 2^{\mathrm{L} 14 \mathrm{P} / \mathrm{L} 14 \mathrm{P}}$ females $(\mathrm{n}=8)$ were sexually active. However, both ovaries $(n=5)$ were small, atrophic and free of identifiable follicles and fibrosis at $90 \mathrm{dpp}$ for $47 \%$ of the infertile females (figure 4B).

For the $53 \%$ fertile $\operatorname{Xrcc}^{\mathrm{L} 14 \mathrm{P} / \mathrm{L} 14 \mathrm{P}}$ females, the litter size was significantly reduced, and an average of 4.5 mice was produced by each litter $(p<0.01)$. The mating time to give birth was evidently prolonged to an average of 44 days $(\mathrm{p}<0.01$; online supplementary table S15). Their ovarian 

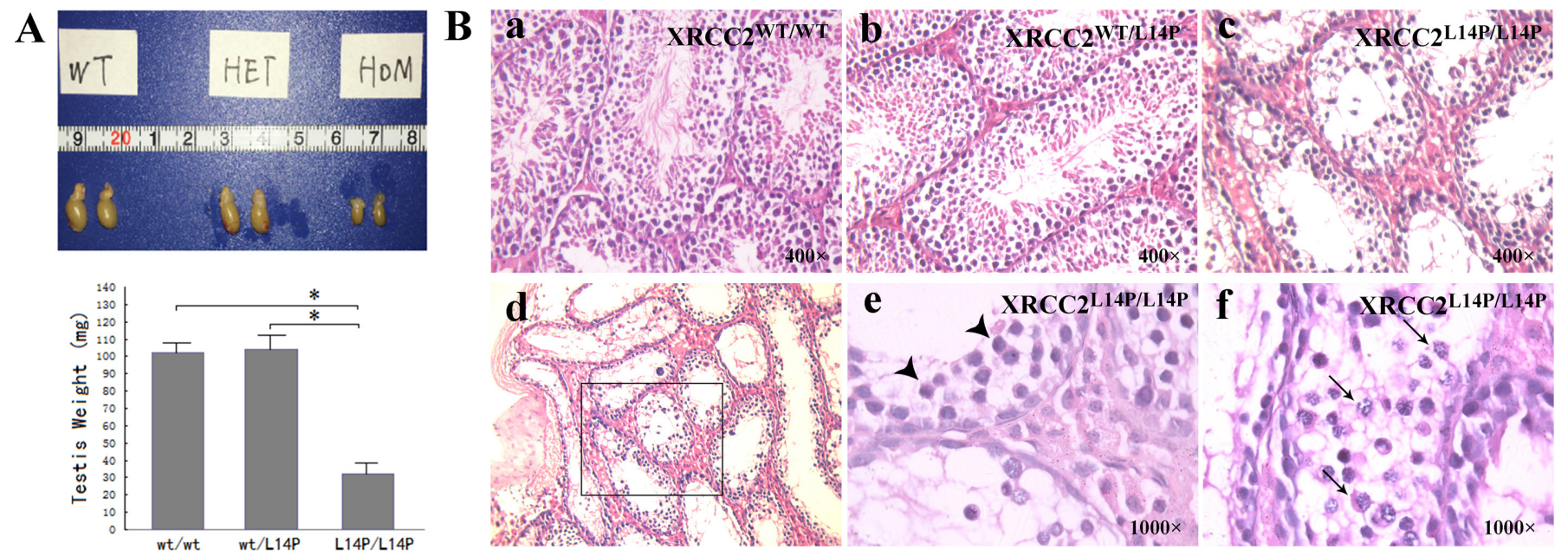

\section{TUNEL staining}
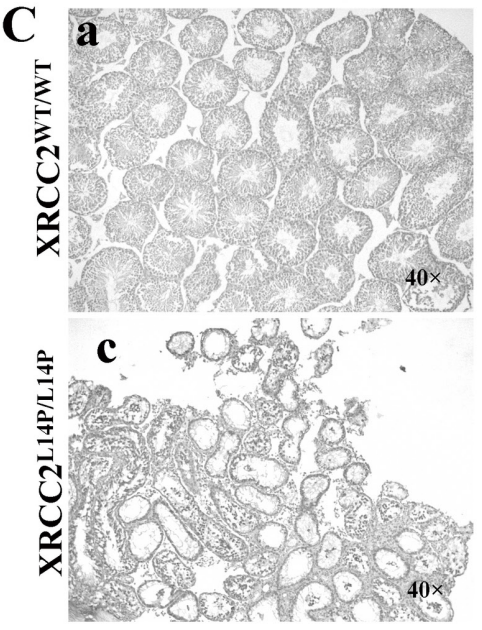

\section{b}
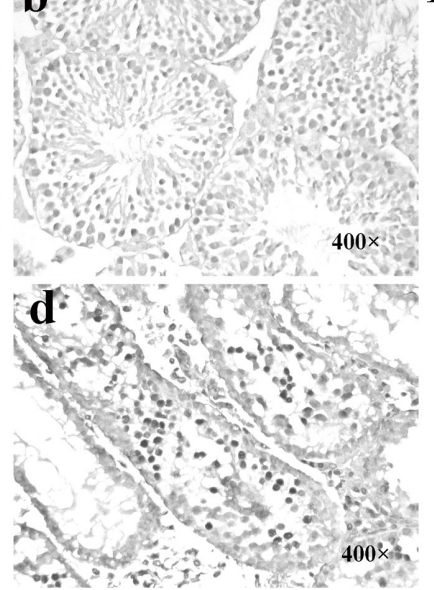

XRCC2 staining
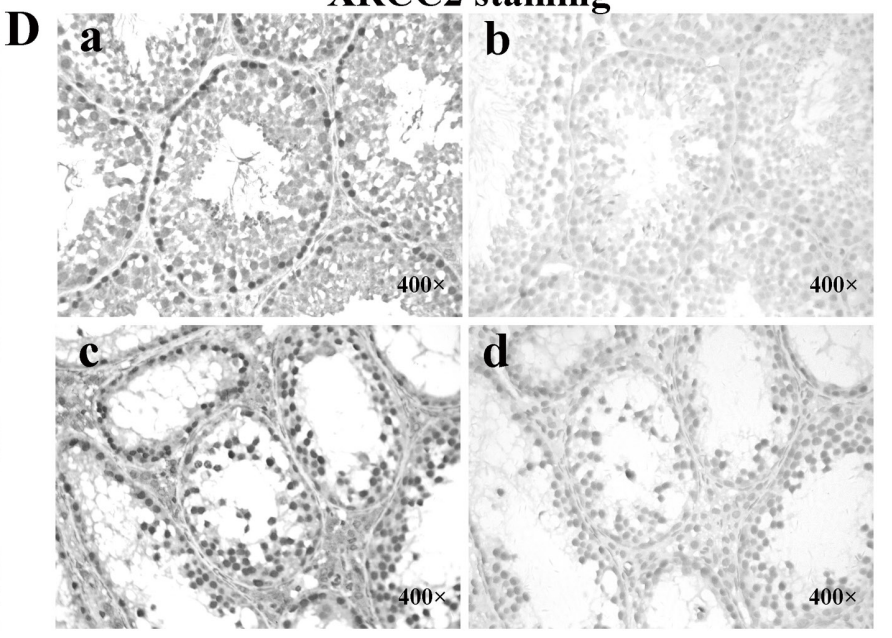

Figure 3 Effects of $X r c c 2^{L 14 P}$ in male mice at 90 days. (A) Testis size (up) and weights (down) for respective genotypes are shown. Error bars indicate SD from the mean of the testis weights $(n=6)$. Asterisks represent statistical significance $(p<0.05)$. (B) H\&E staining of mice testes. (a) tubules of $X r c c{ }^{W T /}$ ${ }^{W T} ;(b)$ tubules of $X_{r c c} 2^{\text {WT/L14P }} ;(c, d, e, f)$ tubules of $X_{r c c} 2^{L 14 P / L 14 P}$, arrowhead: zygotene stage cells; arrow: apoptotic like-pachytene stage cells. Magnification: $a, b, c=400 x ; d=400 x ; e, f=1000 x$. (C) TUNEL staining of $X_{r c c} 2^{\text {WT/WT }}$ and $\operatorname{Xrcc}^{\text {L14P/L14P }}$ testes. Apoptotic cells are labelled in dark brown. (a,b) Xrcc $2^{\text {WT/W }}$ testes showed no apoptotic spermatocytes. (c,d) Apoptotic spermatocytes were found in tubules of Xrcc $2^{L 14 P / L 14 P}$ testes. Magnification: $a, c=40 x ; b, d=400 \times$. (D) Testis sections were immunohistochemically labelled for Xrcc2 (ac) (in dark brown) and section stained with IgG antibody (b,d) as a negative control. $(a, b) \operatorname{Xrcc}^{\text {WT/WT }}$ testis; $(c, d) \operatorname{Xrcc}^{L 14 P / L 14 P}$ testis. Magnification: $a, b, c, d=400 \times$.

abnormalities, including small sizes, atrophy and no identifiable follicles, were prevalent on one side (figure 4C and D).

We further evaluated 60 ovaries from 7 to $180 \mathrm{dpp} \mathrm{Xrcc} 2^{\mathrm{L} 14 \mathrm{P} /}$ ${ }^{\mathrm{L} 14 \mathrm{P}}$ females (online supplementary table S16 and figure S7). The results showed that follicles could be observed in $100 \%$ of 7 or $14 \mathrm{dpp}$ ovaries, $80 \%$ of $21 \mathrm{dpp}$ ovaries, $70 \%$ of 28 dpp ovaries, $50 \%$ of $90 \mathrm{dpp}$ ovaries and $20 \%$ of $180 \mathrm{dpp}$ ovaries (online supplementary table S16 and figure S8). These data indicated premature ovarian failure.

\section{DISCUSSION}

Meiotic HR participates in gametogenesis. In most eukaryotes, meiotic HR is mediated by two recombinase systems, namely, ubiquitous RAD51 and meiosis-specific DMC1. Meiotic HR is initiated by the formation of DNA DSBs that are catalysed by the enzyme SPO11. ${ }^{34-36}$ DNA ends are then resected at DSB sites. Two RecA-related recombinases, namely, DMC1 and RAD51, form nucleoprotein filaments on a single-strand DNA flanking these DSB sites. They catalyse strand invasion and strand exchange reaction with a homologous interval on another DNA molecule to ensure successful repair. Five other RAD51 paralogues forming at least two stable complexes, namely, BCDX2: RAD51B-RAD51C-RAD51D-XRCC2 and CX3: RAD51C-XRCC3, have been reported to play important roles in RAD51-mediated HR-DSB repair. ${ }^{8}$ The BCDX2 complex is responsible for RAD51 recruitment or stabilisation at damage sites, ${ }^{20} 3738$ whereas the CX3 complex acts downstream of RAD51 recruitment to damage sites associated with Holliday junction resolvase activity, possibly indicating its function of stabilising gene conversion tracts. ${ }^{2038}$ DMC1 is a meiosis-specific recombinase, ${ }^{17}$ whereas RAD51 is essential for mitotic and meiotic recombination. ${ }^{18} 19$ This role is consistent with the association of an XRCC2 gene variant or other RAD51 paralogue variants with a high risk of cancer. ${ }^{39}$ Our study did not find any evidence of cancer in the family despite a careful inquiry possibly because the sample size of the family was limited, or the XRCC2 Ler14Pro mutation did not disrupt the HR function of somatic cells.

However, whether the RAD51-HR system uses the same mechanism in mitotic and meiotic cells remains unclear. ${ }^{19}$ In 
A

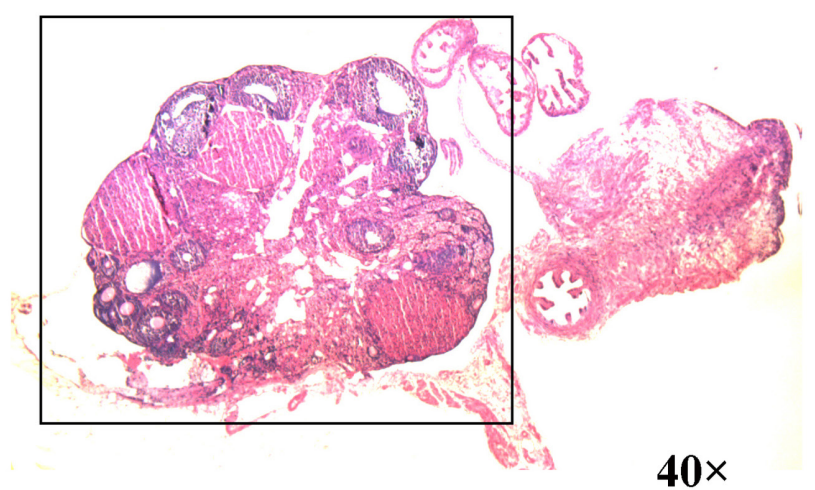

B humans, researchers have yet to determine why two homologous DNA recombinases, namely, RAD51 and DMC1, are required in mammalian meiosis. ${ }^{4041}$ To identify the mechanism of HR, we should create a RAD51 mutant model that specifically disrupts only one of the RAD51-HR-DSB repair functions, which are mitosis and meiosis HR-DSB repair functions. Unfortunately, knockout rodents of Rad51 or Rad51 paralogues are embryonically lethal, ${ }^{20-24}$ so mechanistic studies on RAD51-HR-DSB repair in meiosis are limited. Using a separation-of-function mutant form of Rad51, Veronica Cloud et $a l^{19}$ reported that $\operatorname{Rad} 51$ performs a filament-forming function in HR of meiosis, but the function of homologous joint molecules is unnecessary. This observation probably indicates that Rad51 uses different functions between mitotic and meiotic cells. In the present study, we identified XRCC2 recessive mutations in a family with complete meiotic arrest, azoospermia and infertility. The mouse model with the $X r c c 2^{\mathrm{L} 14 \mathrm{P}}$ mutation replicated the phenotypes. Homozygous female mice exhibited reproductive disorders that were consistent with premature ovary failure. Human XRCC2 and mouse Xrcc2 mutants did not exhibit other identifiable phenotypes. Although Xrcc2-c.41T>C showed 30\% splicing changes, the full $\mathrm{Xrcc} 2$ protein was detected. We then proposed that
XRCC2-Leu14Pro was a meiosis-specific mutation, and this finding was further confirmed by the following aspects.

First, unlike an XRCC2-truncated male showing congenital malformations and atypical Fanconi anaemia, patients with XRCC2-c.41T >C/p.Leu14Pro exhibited infertility only. Shamseldin et $a l^{31}$ and Park et $a l^{32}$ identified a truncating XRCC2 (homozygous XRCC2-p.Arg215*) on a 2.5-year-old male who displayed microcephaly, absent thumbs, absent first metacarpal and scaphoid bones, absent radius, facial paralysis, ectopic kidney and severe growth deficiency. The somatic cells of this child displayed a marked increase in the frequency of unrepaired DSBs in response to crosslinking agents. Thus, the child was proposed to be affected with atypical Fanconi anaemia. ${ }^{3132}$ In the present study, when the infertile brothers (IV:2 and IV:3) with the XRCC2-c.41T >C/p.Leu14Pro homozygous mutation were examined independently by a neurologist, haematologist and orthopaedist, neither exhibited any of the abovementioned malformations (data not shown). In the assay of the somatic cells cultured with DNA breakage agents, the frequencies of the unrepaired chromosomal breaks of XRCC2-p.Leu14Pro lymphocytes did not increase (online supplementary table S7 and figure S5). Thus, the XRCC2-p.Leu14Pro mutation did not disrupt the HR-DSB 
repair function in somatic cells, or the effect was minimal that it was hardly distinguished.

Second, in contrast to the $X r c c 2^{-1-}$ mice exhibiting embryonic lethality, reduced size and morphological abnormalities, the $X r c c 2^{\mathrm{L} 14 \mathrm{P} / \mathrm{L} 14 \mathrm{P}}$ mice were viable with unreduced size and did not show identifiable malformation other than reproductive defects. These observations verified that Xrcc2 is involved in HR-mediated DSB repair. 2223132 Deans et al ${ }^{22}$ constructed an Xrcc2 knockout model, in which exon 3 ( $86 \%$ ofanXrcc 2 coding sequence) is deleted. In more than 300 offspring, heterozygote intercrosses fail to produce any $\mathrm{Xrcc2}^{-/-}$mice, thereby indicating that $\mathrm{Xrcc}^{-/-}$mice are embryonically lethal. ${ }^{22}$ Additionally, $X r c c 2^{-/-}$embryos displaying growth retardation and morphological abnormalities are observed. ${ }^{22}$ In the present study, the $X r c c 2^{\mathrm{L} 14 \mathrm{P}}$ mouse model revealed that the heterozygote intercrosses transmitted the mutation to the offspring at normal Mendelian frequencies (about 1:2:1; supplementary table S8). The Xrcc2 $2^{\text {L14P/L14P }}$ mice were viable, and their size, body weights and physical characteristics were indistinguishable among the three groups of offspring (online supplementary tables S8-S12 and figure S6).

Third, p.Leu14Pro is located on the linker region of XRCC2. In RAD51 paralogues, we observed that all of the members except XRCC2 are composed of three domains, namely, $\mathrm{N}$-terminal and $\mathrm{C}$-terminal domains connected by a linker region ${ }^{41}$ (online supplementary figure S9). However, XRCC2 comprises two domains, namely, a C-terminal domain and a linker region, and lacks an $\mathrm{N}$-terminal domain ${ }^{41}$ (online supplementary figure S9). Previous studies stated that the linker region of RAD51 paralogues is essential for protein-protein interactions, ${ }^{20} 42$ and two complexes, namely, BCDX2 and CX3, have been suggested. ${ }^{20} 3738$ In the present study, p.Leu14Pro mutation occurred on the linker region of XRCC2 (online supplementary figure S9), and this phenomenon is different from the previously reported truncating p.Arg $215 \%$ mutation that truncated a part of the C-terminal region of XRCC2 (online supplementary figure S8A) on a patient with atypical Fanconi anaemia. 3032

Therefore, this study identified a novel genetic entity of HR-mediated DNA repair gene mutation, that is, the point mutation on the linker region of XRCC2 was a meiosis-specific mutation causing meiotic arrest and infertility. Further functional studies on the mutation in the XRCC2 linker region should be conducted.

Acknowledgements The authors are grateful to the family members who participated in this study.

Contributors YJY and LQW designed the research. YJY, JHG, LD, YMZ, HH, LHT, WJC, JHG, MT, KWW performed the sample collection and the research. YJY, JHG and LD performed the bioinformatics analysis. YJY and LQW analysed the experimental data and wrote the paper.

Funding This work was supported by grants from the National Natural Science Foundation of China (31501017, to YJY), the Key Nature Science Foundation of Hunan Children's Hospital (2015-0002, to YJY), the National Natural Science Foundation of China (81771599, to LQW), and the Key Laboratory fund of Hunan Province (2018TP1028, to YYJ).

Competing interests None declared.

Patient consent Obtained.

Ethics approval Hunan Children's Hospital.

Provenance and peer review Not commissioned; externally peer reviewed.

Open access This is an open access article distributed in accordance with the Creative Commons Attribution Non Commercial (CC BY-NC 4.0) license, which permits others to distribute, remix, adapt, build upon this work non-commercially, and license their derivative works on different terms, provided the original work is properly cited, appropriate credit is given, any changes made indicated, and the use is non-commercial. See: http://creativecommons.org/licenses/by-nc/4.0/.

\section{REFERENCES}

1 Lotti F, Maggi M. Ultrasound of the male genital tract in relation to male reproductive health. Hum Reprod Update 2015;21:56-83

2 American Urological Association. The evaluation of the azoospermic male: AUA best practice statement. $2011 \mathrm{https}$ ://www.auanet.org/guidelines/male-infertilityazoospermic-male-(reviewed-and-amended-2011).

3 Matzuk MM, Lamb DJ. The biology of infertility: research advances and clinical challenges. Nat Med 2008;14:1197-213.

4 Lee JY, Dada R, Sabanegh E, Carpi A, Agarwal A. Role of genetics in azoospermia. Urology 2011;77:598-601.

5 Miyamoto T, Hasuike S, Yogev L, Maduro MR, Ishikawa M, Westphal H, Lamb DJ. Azoospermia in patients heterozygous for a mutation in SYCP3. Lancet 2003:362:1714-9.

6 Bashamboo A, Ferraz-de-Souza B, Lourenço D, Lin L, Sebire NJ, Montjean D, BignonTopalovic J, Mandelbaum J, Siffroi JP, Christin-Maitre S, Radhakrishna U, Rouba H, Ravel C, Seeler J, Achermann JC, McElreavey K. Human male infertility associated with mutations in NR5A1 encoding steroidogenic factor 1. Am J Hum Genet 2010;87:505-12

7 Arafat M, Har-Vardi I, Harlev A, Levitas E, Zeadna A, Abofoul-Azab M, Dyomin V, Sheffield VC, Lunenfeld E, Huleihel M, Parvari R. Mutation in TDRD9 causes nonobstructive azoospermia in infertile men. J Med Genet 2017;54:633-9.

8 Ayhan Ö, Balkan M, Guven A, Hazan R, Atar M, Tok A, Tolun A. Truncating mutations in TAF4B and ZMYND15 causing recessive azoospermia. J Med Genet 2014:51:239-44.

9 Yatsenko AN, Georgiadis AP, Röpke A, Berman AJ, Jaffe T, Olszewska M, Westernströer B, Sanfilippo J, Kurpisz M, Rajkovic A, Yatsenko SA, Kliesch S, Schlatt S, Tüttelmann F. $\mathrm{X}$-linked TEX11 mutations, meiotic arrest, and azoospermia in infertile men. $N$ Engl J Med 2015:372:2097-107.

10 Yang F, Silber S, Leu NA, Oates RD, Marszalek JD, Skaletsky H, Brown LG, Rozen S, Page DC, Wang PJ. TEX11 is mutated in infertile men with azoospermia and regulates genome-wide recombination rates in mouse. EMBO Mol Med 2015:7:1198-210.

11 He WB, Tu CF, Liu Q, Meng LL, Yuan SM, Luo AX, He FS, Shen J, Li W, Du J, Zhong CG, Lu GX, Lin G, Fan LQ, Tan YQ. DMC1 mutation that causes human non-obstructive azoospermia and premature ovarian insufficiency identified by whole-exome sequencing. J Med Genet 2018;55:198-204

12 Tan YQ, Tu C, Meng L, Yuan S, Sjaarda C, Luo A, Du J, Li W, Gong F, Zhong C, Deng HX, Lu G, Liang P, Lin G. Loss-of-function mutations in TDRD7 lead to a rare novel syndrome combining congenital cataract and nonobstructive azoospermia in humans. Genet Med 2017

13 Okutman O, Muller J, Skory V, Garnier JM, Gaucherot A, Baert Y, Lamour V, Serdarogullari M, Gultomruk M, Röpke A, Kliesch S, Herbepin V, Aknin I, Benkhalifa M Teletin M, Bakircioglu E, Goossens E, Charlet-Berguerand N, Bahceci M, Tüttelmann $\mathrm{F}$, Viville $\mathrm{S}$. A no-stop mutation in MAGEB4 is a possible cause of rare $\mathrm{X}$-linked azoospermia and oligozoospermia in a consanguineous Turkish family. J Assist Reprod Genet 2017:34:683-94.

14 Maor-Sagie E, Cinnamon Y, Yaacov B, Shaag A, Goldsmidt H, Zenvirt S, Laufer N, Richler C, Frumkin A. Deleterious mutation in SYCE1 is associated with nonobstructive azoospermia. J Assist Reprod Genet 2015;32:887-91.

15 Choi Y, Jeon S, Choi M, Lee MH, Park M, Lee DR, Jun KY, Kwon Y, Lee OH, Song SH, Kim JY, Lee KA, Yoon TK, Rajkovic A, Shim SH. Mutations in SOHLH1 gene associate with nonobstructive azoospermia. Hum Mutat 2010;31:788-93.

16 Tenenbaum-Rakover Y, Weinberg-Shukron A, Renbaum P, Lobel O, Eideh H, Gulsuner S, Dahary D, Abu-Rayyan A, Kanaan M, Levy-Lahad E, Bercovich D, Zangen D. Minichromosome maintenance complex component 8 (MCM8) gene mutations result in primary gonadal failure. J Med Genet 2015;52:391-9.

17 Bishop DK, Park D, Xu L, Kleckner N. DMC1: a meiosis-specific yeast homolog of E. coli recA required for recombination, synaptonemal complex formation, and cell cycle progression. Cell 1992;69:439-56.

18 Zadorozhny K, Sannino V, Beláň O, Mlčoušková J, Špírek M, Costanzo V, Krejčí L. Fanconi-anemia-associated mutations destabilize RAD51 filaments and impair replication fork protection. Cell Rep 2017;21:333-40.

19 Cloud V, Chan YL, Grubb J, Budke B, Bishop DK. Rad51 is an accessory factor for Dmc1-mediated joint molecule formation during meiosis. Science 2012:337:1222-5.

20 Chun J, Buechelmaier ES, Powell SN. Rad51 paralog complexes BCDX2 and CX3 act at different stages in the BRCA1-BRCA2-dependent homologous recombination pathway. Mol Cell Biol 2013;33:387-95.

21 Shu Z, Smith S, Wang L, Rice MC, Kmiec EB. Disruption of muREC2/RAD51L1 in mice results in early embryonic lethality which can Be partially rescued in a $\mathrm{p53}(-/-)$ background. Mol Cell Biol 1999;19:8686-93.

22 Deans B, Griffin CS, Maconochie M, Thacker J. Xrcc2 is required for genetic stability, embryonic neurogenesis and viability in mice. Embo J 2000;19:6675-85.

23 Pittman DL, Schimenti JC. Midgestation lethality in mice deficient for the RecA-related gene, Rad51d/Rad5113. Genesis 2000;26:167-73. 
24 Johnson RD, Liu N, Jasin M. Mammalian XRCC2 promotes the repair of DNA doublestrand breaks by homologous recombination. Nature 1999;401:397-9.

25 Lim DS, Hasty P. A mutation in mouse rad51 results in an early embryonic lethal that is suppressed by a mutation in p53. Mol Cell Biol 1996;16:7133-43.

26 Li R, Yu C, Li Y, Lam TW, Yiu SM, Kristiansen K, Wang J. SOAP2: an improved ultrafast tool for short read alignment. Bioinformatics 2009;25:1966-7.

27 Li R, Li Y, Fang X, Yang H, Wang J, Kristiansen K, Wang J. SNP detection for massively parallel whole-genome resequencing. Genome Res 2009;19:1124-32.

$28 \mathrm{Li} \mathrm{H}$, Durbin R. Fast and accurate long-read alignment with Burrows-Wheeler transform. Bioinformatics 2010;26:589-95.

29 McKenna A, Hanna M, Banks E, Sivachenko A, Cibulskis K, Kernytsky A, Garimella K, Altshuler D, Gabriel S, Daly M, DePristo MA. The Genome Analysis Toolkit: a MapReduce framework for analyzing next-generation DNA sequencing data. Genome Res 2010;20:1297-303.

30 Li Q, Wang K. InterVar: clinical interpretation of genetic variants by the 2015 ACMGAMP Guidelines. Am J Hum Genet 2017;100:267-80.

31 Shamseldin HE, Elfaki M, Alkuraya FS. Exome sequencing reveals a novel Fanconi group defined by XRCC2 mutation. J Med Genet 2012;49:184-6.

32 Park JY, Virts EL, Jankowska A, Wiek C, Othman M, Chakraborty SC, Vance GH, Alkuraya FS, Hanenberg $\mathrm{H}$, Andreassen PR. Complementation of hypersensitivity to DNA interstrand crosslinking agents demonstrates that XRCC2 is a Fanconi anaemia gene. J Med Genet 2016;53:672-80.

33 Cartwright R, Tambini CE, Simpson PJ, Thacker J. The XRCC2 DNA repair gene from human and mouse encodes a novel member of the recA/RAD51 family. Nucleic Acids Res 1998;26:3084-9.

34 Baudat F, Manova K, Yuen JP, Jasin M, Keeney S. Chromosome synapsis defects and sexually dimorphic meiotic progression in mice lacking Spo11. Mol Cell 2000;6:989-98.
35 Keeney S, Giroux CN, Kleckner N. Meiosis-specific DNA double-strand breaks are catalyzed by Spo11, a member of a widely conserved protein family. Cell 1997;88:375-84.

36 Romanienko PJ, Camerini-Otero RD. The mouse Spo11 gene is required for meiotic chromosome synapsis. Mol Cell 2000;6:975-87.

37 Masson JY, Tarsounas MC, Stasiak AZ, Stasiak A, Shah R, Mcllwraith MJ, Benson FE, West SC. Identification and purification of two distinct complexes containing the five RAD51 paralogs. Genes Dev 2001;15:3296-307.

38 Masson JY, Stasiak AZ, Stasiak A, Benson FE, West SC. Complex formation by the human RAD51C and XRCC3 recombination repair proteins. Proc Natl Acad Sci U SA 2001;98:8440-6.

39 Park DJ, Lesueur F, Nguyen-Dumont T, Pertesi M, Odefrey F, Hammet F, Neuhausen SL, John EM, Andrulis IL, Terry MB, Daly M, Buys S, Le Calvez-Kelm F, Lonie A, Pope BJ, Tsimiklis H, Voegele C, Hilbers FM, Hoogerbrugge N, Barroso A, Osorio A, Giles GG, Devilee P, Benitez J, Hopper JL, Tavtigian SV, Goldgar DE, Southey MC. Breast Cancer Family RegistryKathleen Cuningham Foundation Consortium for Research into Familia Breast Cancer. Rare mutations in XRCC2 increase the risk of breast cancer. Am J Hum Genet 2012;90:734-9.

40 Dai J, Voloshin 0, Potapova S, Camerini-Otero RD. Meiotic knockdown and complementation reveals essential role of RAD51 in mouse spermatogenesis. Cell Rep 2017;18:1383-94.

41 Kuznetsov S, Pellegrini M, Shuda K, Fernandez-Capetillo O, Liu Y, Martin BK, Burkett S, Southon E, Pati D, Tessarollo L, West SC, Donovan PJ, Nussenzweig A, Sharan SK. RAD51C deficiency in mice results in early prophase I arrest in males and sister chromatid separation at metaphase II in females. J Cell Biol 2007;176:581-92.

42 Miller KA, Sawicka D, Barsky D, Albala JS. Domain mapping of the Rad51 paralog protein complexes. Nucleic Acids Res 2004;32:169-78. 